\title{
An Analysis of Differentially Expressed Coding and Long Non-Coding RNAs in Multiple Models of Skeletal Muscle Atrophy
}

\author{
Keisuke Hitachi ${ }^{1}$ (D), Masashi Nakatani ${ }^{1,2}$, Yuri Kiyofuji ${ }^{1}$, Hidehito Inagaki ${ }^{3,4}$, Hiroki Kurahashi ${ }^{3,4}$ and \\ Kunihiro Tsuchida ${ }^{1, *(D)}$ \\ 1 Division for Therapies against Intractable Diseases, Institute for Comprehensive Medical Science (ICMS), \\ Fujita Health University, Toyoake 470-1192, Japan; hkeisuke@fujita-hu.ac.jp (K.H.); \\ nakatani@seijoh-u.ac.jp (M.N.); nanbyou@fujita-hu.ac.jp (Y.K.) \\ 2 Faculty of Rehabilitation and Care, Seijoh University, Tokai 476-0014, Japan \\ 3 Genome and Transcriptome Analysis Center, Fujita Health University, Toyoake 470-1192, Japan; \\ hinagaki@fujita-hu.ac.jp (H.I.); kura@fujita-hu.ac.jp (H.K.) \\ 4 Division of Molecular Genetics, Institute for Comprehensive Medical Science (ICMS), \\ Fujita Health University, Toyoake 470-1192, Japan \\ * Correspondence: tsuchida@fujita-hu.ac.jp; Tel.: +81-(562)-93-9384
}

check for updates

Citation: Hitachi, K.; Nakatani, M.; Kiyofuji, Y.; Inagaki, H.; Kurahashi, H.; Tsuchida, K. An Analysis of Differentially Expressed Coding and Long Non-Coding RNAs in Multiple Models of Skeletal Muscle Atrophy. Int. J. Mol. Sci. 2021, 22, 2558. https://doi.org/10.3390/ ijms22052558

Academic Editor: Nicoletta Potenza

Received: 15 February 2021

Accepted: 26 February 2021

Published: 4 March 2021

Publisher's Note: MDPI stays neutral with regard to jurisdictional claims in published maps and institutional affiliations.

Copyright: (c) 2021 by the authors. Licensee MDPI, Basel, Switzerland. This article is an open access article distributed under the terms and conditions of the Creative Commons Attribution (CC BY) license (https:/ / creativecommons.org/licenses/by/ $4.0 /)$.

\begin{abstract}
The loss of skeletal muscle mass (muscle atrophy or wasting) caused by aging, diseases, and injury decreases quality of life, survival rates, and healthy life expectancy in humans. Although long non-coding RNAs (lncRNAs) have been implicated in skeletal muscle formation and differentiation, their precise roles in muscle atrophy remain unclear. In this study, we used RNA-sequencing (RNASeq) to examine changes in the expression of lncRNAs in four muscle atrophy conditions (denervation, casting, fasting, and cancer cachexia) in mice. We successfully identified 33 annotated lncRNAs and 18 novel lncRNAs with common expression changes in all four muscle atrophy conditions. Furthermore, an analysis of lncRNA-mRNA correlations revealed that several lncRNAs affected small molecule biosynthetic processes during muscle atrophy. These results provide novel insights into the lncRNA-mediated regulatory mechanism underlying muscle atrophy and may be useful for the identification of promising therapeutic targets.
\end{abstract}

Keywords: long non-coding RNA; mRNA; skeletal muscle atrophy; RNA-sequencing

\section{Introduction}

In the last decade, increasing evidence has indicated that long non-coding RNAs (lncRNAs), defined as having more than 200 bases in length, have important roles in cell proliferation and differentiation, development, and the maintenance of homeostasis via interactions with essential proteins [1]. According to the most recent update of the LncBook database, there are 268,848 lncRNA genes in humans [2]. This is more than ten times the 19,972 protein-coding genes in the GENCODE database [3] and more than an order of magnitude above the number of known microRNAs [4], which are small non-coding RNAs of 16-28 bases in length. In addition, lncRNA mutations and dysregulation are associated with the development and progression of human diseases, including cancer, cardiovascular diseases, and neurodegenerative diseases [5-7].

Skeletal muscle is a mechanically pivotal organ in humans; it is required for movement of body parts and the body as a whole. Skeletal muscle is also a highly plastic organ, and its mass decreases through aging, immobilization, malnutrition, and diseases, including cancer, cardiovascular diseases, and neurodegenerative diseases [8-13]. This decrease in muscle mass is referred to as skeletal muscle atrophy and is a cause of decreased activities of daily living and increased mortality from diseases. Muscle atrophy is induced by myostatin, NF-K $\beta$, and glucocorticoid signaling, which activate the ubiquitin-proteasome and 
autophagy-lysosome systems to increase muscle protein breakdown [14-16]. In contrast, insulin-like growth factor-1 (IGF-1)/Akt/mammalian target of rapamycin (mTOR) and $\beta$-adrenergic pathways inhibit muscle atrophy by promoting muscle protein synthesis [17]. The recent discoveries of microRNAs have provided novel insights into the regulation of skeletal muscle mass [18]. In addition, several lncRNAs associated with muscle atrophy have been identified. For example, increased expression of the lncRNA Atrolnc-1 in catabolic conditions promotes muscle atrophy by increasing NF- $\mathrm{kB}$ activity [19]. In a mouse model of amyotrophic lateral sclerosis, the lncRNA Pvt1 is associated with muscle atrophy [20]. We have also found that Myoparr, which is expressed from the promoter region of the myogenin gene, promotes muscle atrophy after denervation treatment by activating myogenin expression and inhibiting BMP signaling. The inhibition of Myoparr attenuates the decrease in muscle mass caused by denervation [21,22]. Thus, the identification and functional characterization of novel lncRNAs associated with muscle atrophy is promising for the development of new therapies.

We have recently shown that muscle atrophy conditions can be classified into two subgroups (disuse-mediated atrophy and systemic wasting atrophy) based on the expression levels of skeletal muscle differentiation-related lncRNAs (DRR, DUM1, linc-MD1, linc- $Y Y 1$, LncMyod, Neat1, Myoparr, Malat1, and SRA) and genomic imprinting-related lncRNAs (Gtl2, H19, and IG-DMR) [23]. It is highly likely that lncRNAs showing commonly altered expression patterns in multiple muscle atrophy conditions are involved in the development or progression of muscle atrophy. However, studies of lncRNAs that are commonly altered across multiple muscle atrophy conditions are lacking [23,24]. In the present study, to identify lncRNAs involved in muscle atrophy, we performed an RNA-sequencing (RNASeq) analysis of lncRNAs using four different muscle atrophy models (denervation, casting, fasting, and cancer cachexia). We detected lncRNAs related to each condition as well as those showing changes of expression in all muscle atrophy conditions. A co-expression network analysis of lncRNAs and mRNAs provided further insights into the biological processes associated with muscle atrophy. These findings improve our understanding of the molecular mechanisms underlying lncRNA-mediated skeletal muscle atrophy and are expected to contribute to the development of novel therapies.

\section{Results}

\section{1. $m R N A$ and $\operatorname{lnc} R N A$ Expression Changes during Skeletal Muscle Atrophy}

We performed an RNA-Seq analysis to identify protein-coding mRNAs and lncRNAs whose expression was altered across multiple skeletal muscle atrophy conditions in mice (denervation, casting, fasting, and cancer-induced cachexia). Based on the expression profiles of both mRNAs and lncRNAs revealed by RNA-Seq, muscle atrophy conditions could be classified into two sub-groups: disuse-mediated atrophy, including the denervation and casting treatments, and systemic wasting atrophy, including fasting and cancer cachexia treatments (Figure 1A). These results were consistent with the expression profiling of individual lncRNAs in our previous report [23]. We identified 895, 1288, 1505, and 1483 up-regulated and 831, 574, 1393, and 1863 down-regulated mRNAs in muscle atrophy conditions caused by denervation, casting, fasting, and cachexia treatments, respectively (Figure 1B and Table S1). In addition, we identified 88, 71, 65, and 127 up-regulated annotated lncRNAs; 110, 86, 119, and 221 down-regulated annotated lncRNAs; 43, 45, 47, and 104 up-regulated unannotated lncRNAs; and 42, 32, 42, and 72 down-regulated unannotated lncRNAs by denervation, casting, fasting, and cachexia treatments, respectively (Figure 1C and Table S1). In this study, annotated and unannotated lncRNAs were defined as sequences registered in any database and the previously unreported sequences, respectively. Forty-four percent of the identified lncRNAs showing altered expression in each muscle atrophy condition overlapped with exons of protein-coding genes or antisense lncRNAs against protein-coding genes (Figure 1D). Additionally, $32 \%$ and $24 \%$ of the identified lncRNAs were located in intergenic and intragenic regions, respectively 
(Figure 1D). Of note, all of the unannotated lncRNAs were found in intergenic regions (Table S1).

A

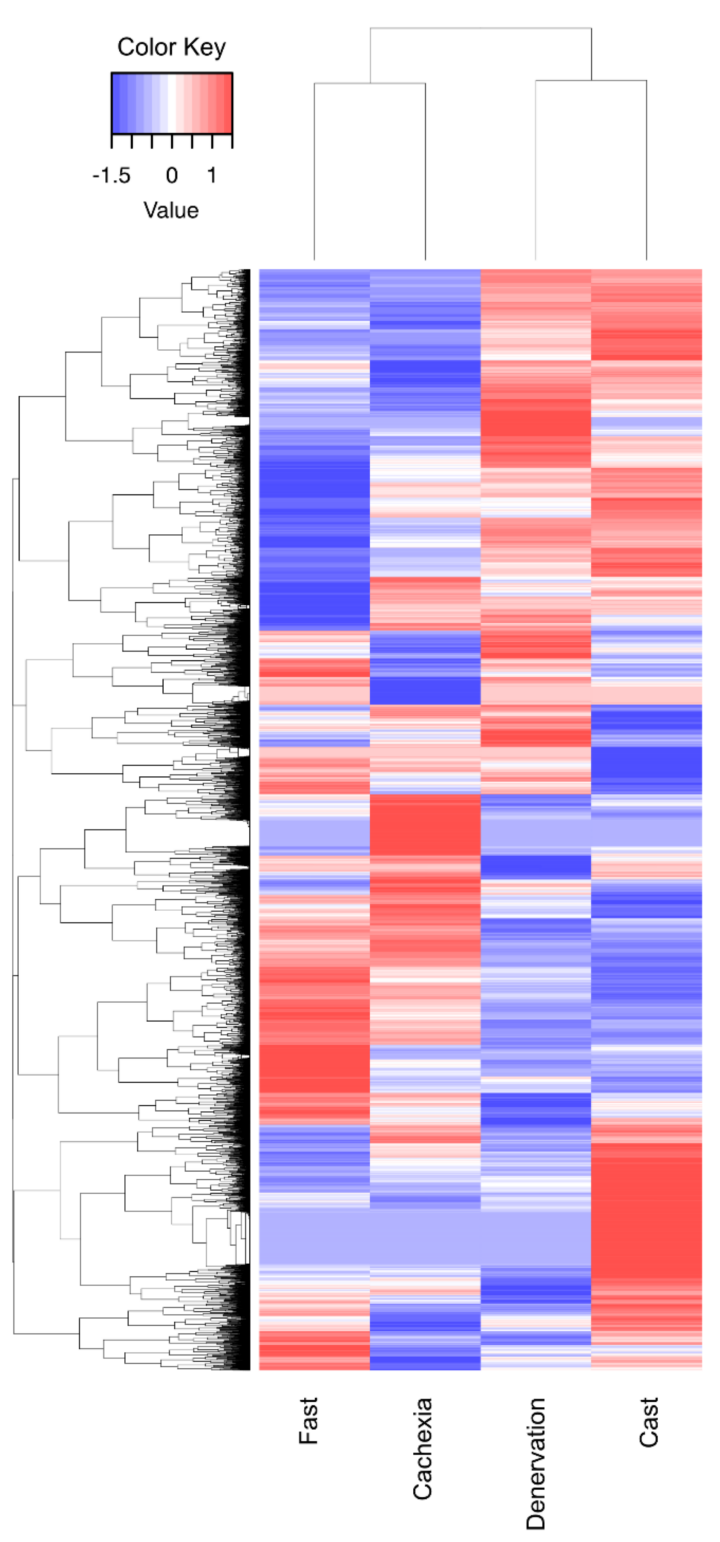

B

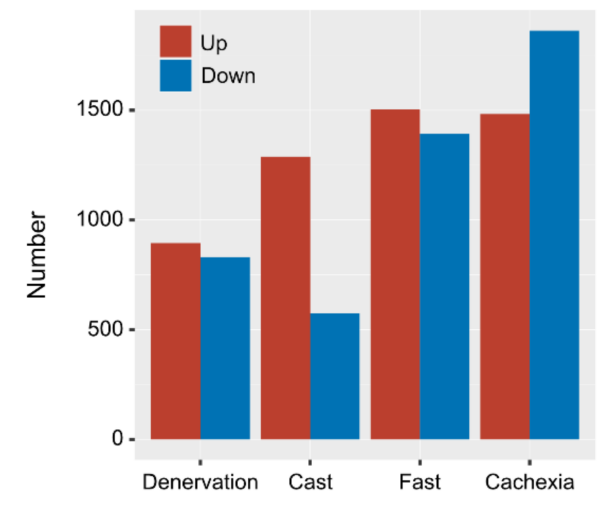

C

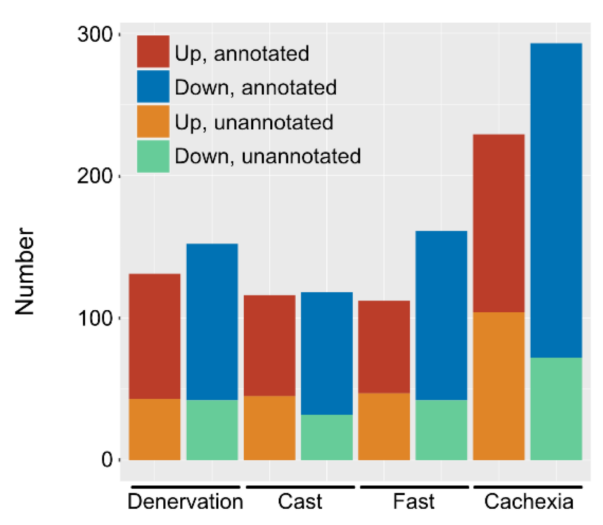

$\mathrm{D}$

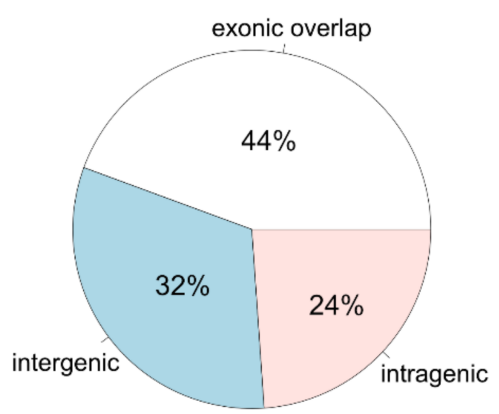

Figure 1. Changes in the expression levels of mRNAs and long non-coding RNAs (lncRNAs) in skeletal muscle atrophy conditions. (A) Hierarchical clustering analysis of differentially expressed genes, including both mRNAs and lncRNAs, after the induction of each atrophy model. (B) Numbers of up- and down-regulated mRNAs in each muscle atrophy condition. Red and blue indicate the up- or down-regulated mRNAs, respectively. (C) Numbers of up- or down-regulated lncRNAs after each treatment. Red, blue, orange, and green indicate up-regulated annotated lncRNAs, down-regulated annotated IncRNAs, up-regulated unannotated lncRNAs, and down-regulated unannotated lncRNAs, respectively. (D) Genomic positions of differentially expressed lncRNAs.

To further characterize the identified mRNAs and lncRNAs, their structural features were examined. The chromosomal distribution of the identified mRNAs and lncRNAs did not differ significantly, except that only lncRNAs were distributed on chromosome $\mathrm{Y}$ and mitochondria (Figure 2A). The lncRNAs typically contained two to four exons, while more than $30 \%$ of the mRNAs contained $>10$ exons (Figure $2 \mathrm{~B}$ ). The mean length of 1 ncRNAs was shorter than that of mRNAs, possibly due to the lower number of exons (Figure 2C). 
The lengths of the open reading frames (ORFs) of the lncRNAs were shorter than those of the mRNAs (Figure 2D).

A

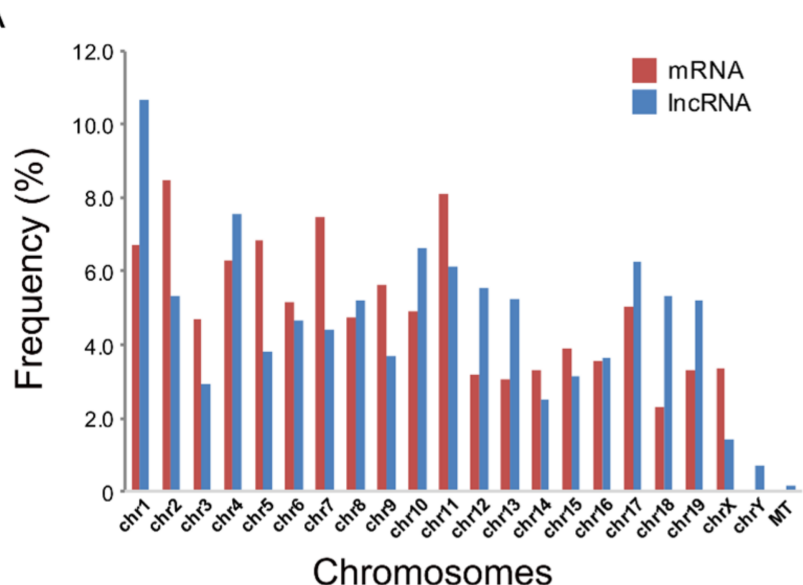

C

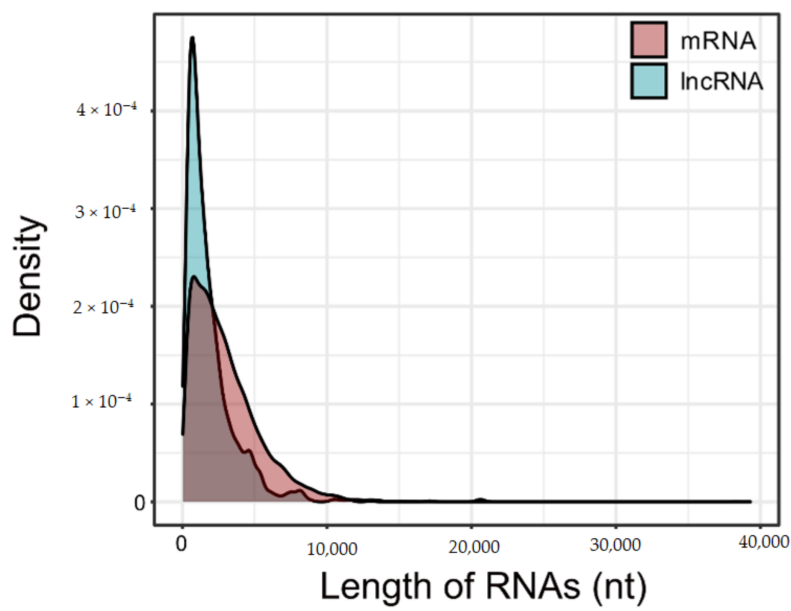

B

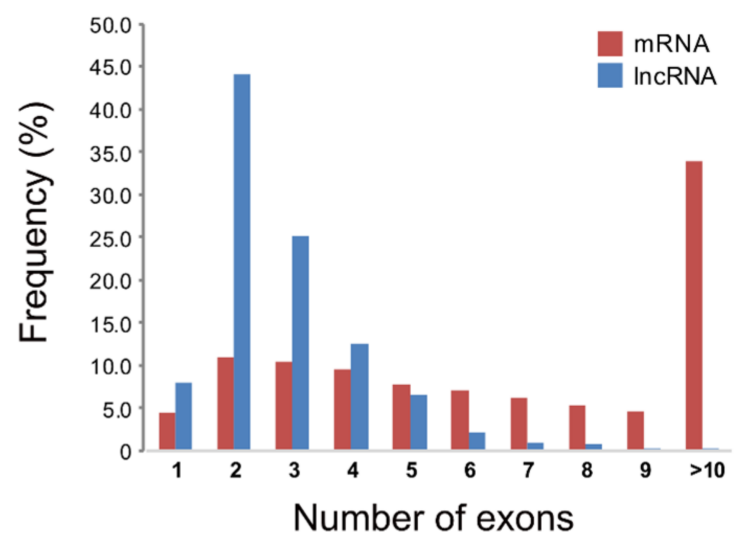

D

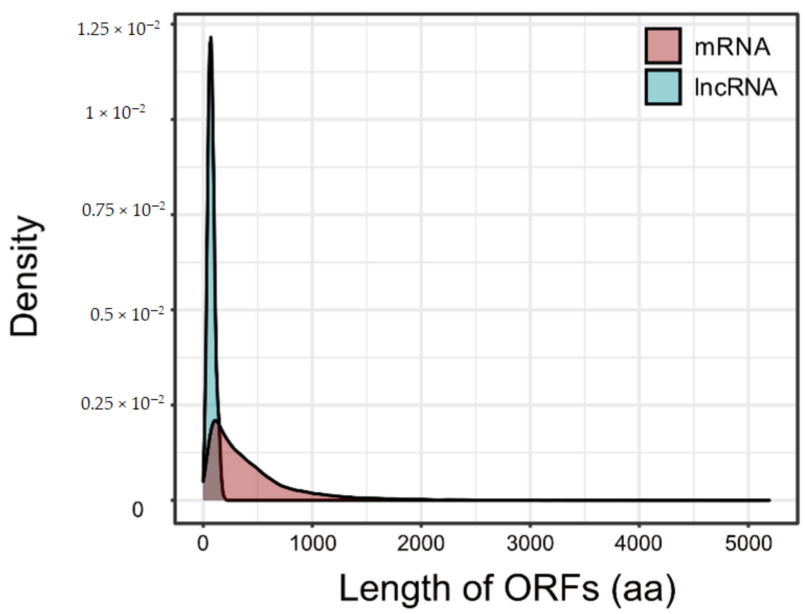

Figure 2. Comparison of sequence features of differentially expressed mRNAs and lncRNAs in muscle atrophy conditions. (A) Genome-wide distribution of differentially expressed mRNAs (red) and lncRNAs (blue) on mouse chromosomes; MT, mitochondria. (B) Frequency of numbers of exons in differentially expressed mRNAs and lncRNAs. (C) Transcript length distribution of differentially expressed mRNAs and lncRNAs; nt: nucleotide. (D) Distribution of open reading frame (ORF) lengths of differentially expressed mRNAs and lncRNAs; aa: amino acids.

\subsection{Functional Characterization of Altered mRNAs in Skeletal Muscle Atrophy}

Next, to clarify the signaling pathways involved in each type of muscle atrophy, we examined the identified mRNAs using a functional enrichment analysis. The upregulated mRNAs, in response to denervation, were preferentially related to rRNA processing, whereas mRNAs that were down-regulated in denervated muscles were enriched in muscle system processes (Figure 3A,B). The up- or down-regulated mRNAs, after casting treatment, were enriched in the inflammatory response or generation of precursor metabolites and energy categories, respectively (Figure 3C,D). Fasting treatment increased the expression levels of mRNAs related to the modification-dependent protein catabolic processes, protein modification by small protein conjugation or removal, and interleukin-1 signaling (Figure 3E). The expression levels of mRNAs related to collagen biosynthesis and modifying enzymes decreased by fasting treatment (Figure 3F). The mRNAs upregulated by cachexia were enriched in the metabolism of RNA and ribonucleoprotein complex biogenesis categories (Figure 3G). The mRNAs involved in the citric acid cycle and respiratory 
electron transport were down-regulated in atrophied muscle caused by cancer cachexia (Figure $3 \mathrm{H}$ ). These results indicated that the primary activated or repressed signaling pathways differed among the muscle atrophy conditions.

A Up-regulated mRNAs by denervation
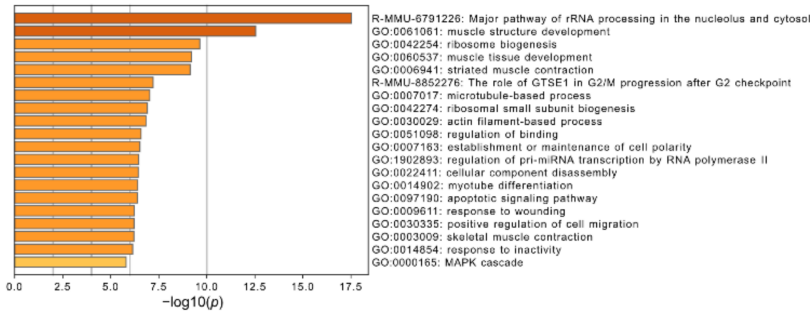

C

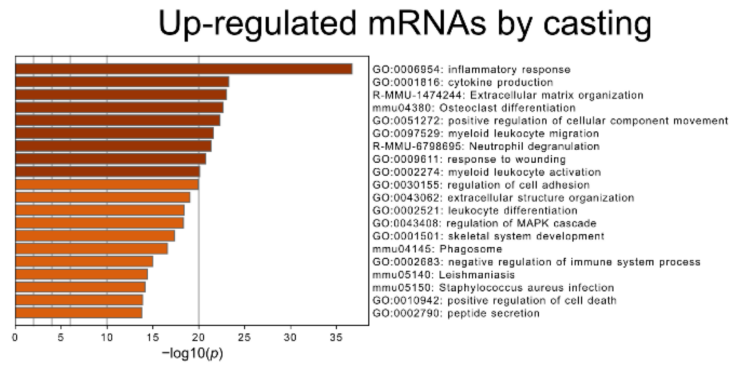

$\mathrm{E}$

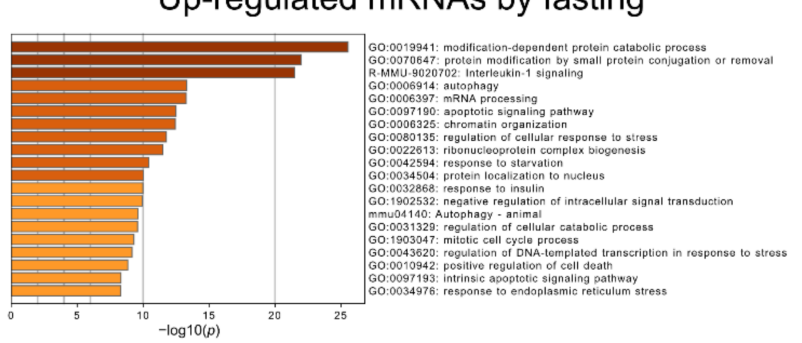

G

Up-regulated mRNAs by cachexia

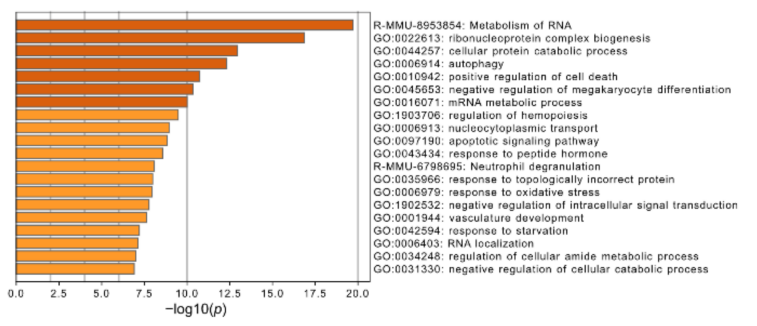

B Down-regulated mRNAs by denervation
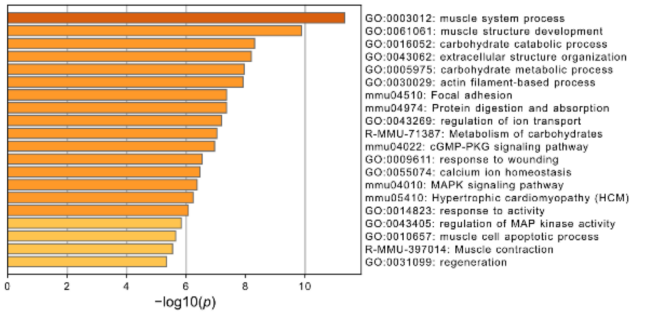

D

Down-regulated mRNAs by casting

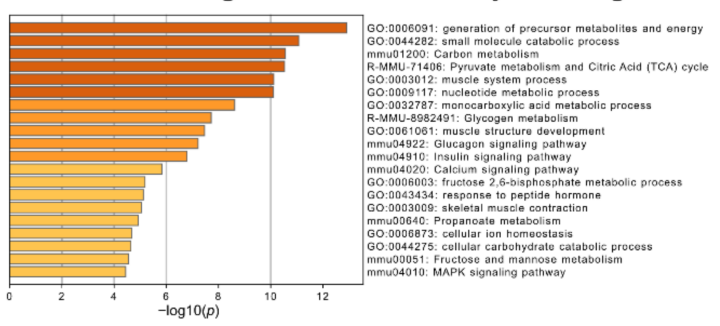

$\mathrm{F}$

Down-regulated mRNAs by fasting

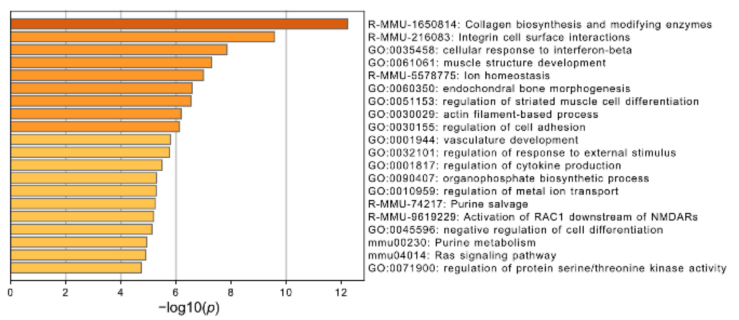

H Down-regulated mRNAs by cachexia

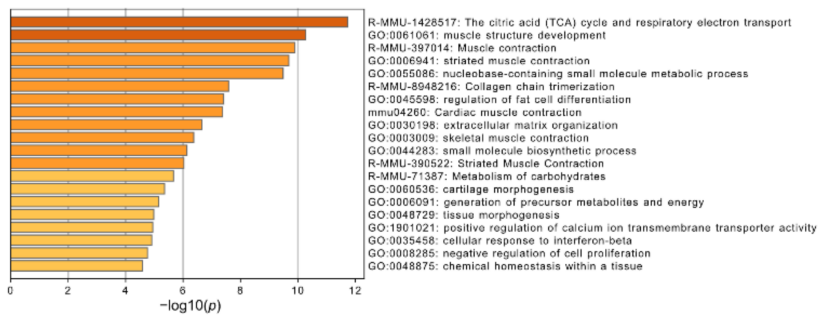

Figure 3. Functional enrichment analysis of differentially expressed mRNAs in each muscle atrophy condition. Enriched biological processes were ranked by $p$-value. Bar graphs show the top, non-redundant enrichment clusters (Metascape analysis) for up- or down-regulated mRNAs in response to denervation (A,B), casting (C,D), fasting (E,F), and cancer cachexia treatment $(\mathbf{G}, \mathbf{H})$. The $x$-axis represents the $-\log 10$ ( $p$-value).

It is likely that mRNAs with altered expression across all four muscle atrophy conditions are involved in the development or progression of skeletal muscle atrophy. We identified 71 and 122 mRNAs whose expression levels were commonly increased and decreased in all muscle atrophy conditions, respectively (Figure 4A,B and Table S2). We evaluated these common mRNAs using a functional enrichment analysis. Interestingly, upregulated mRNAs under the four muscle atrophy conditions were related to the negative regulation of leukocyte activation and regulation of myotube differentiation pathways (Figure 4C). These pathways include genes identified as negative (Cdkn1a and Ctsl) $[25,26]$ 
and positive (Abcc8, Csrp3, Dlg5, Gdf5, Gpnmb, Il4ra, and Runx1) [27-34] regulators of muscle atrophy, supporting the validity of our analysis. Consistent with previous studies of each muscle atrophy condition [35-39], mRNAs with decreased expression in all muscle atrophy conditions were related to the terms of metabolism of carbohydrates and spermine metabolic processes (Figure 4D). Thus, these signaling pathways may be involved in the development or progression of skeletal muscle atrophy.

A

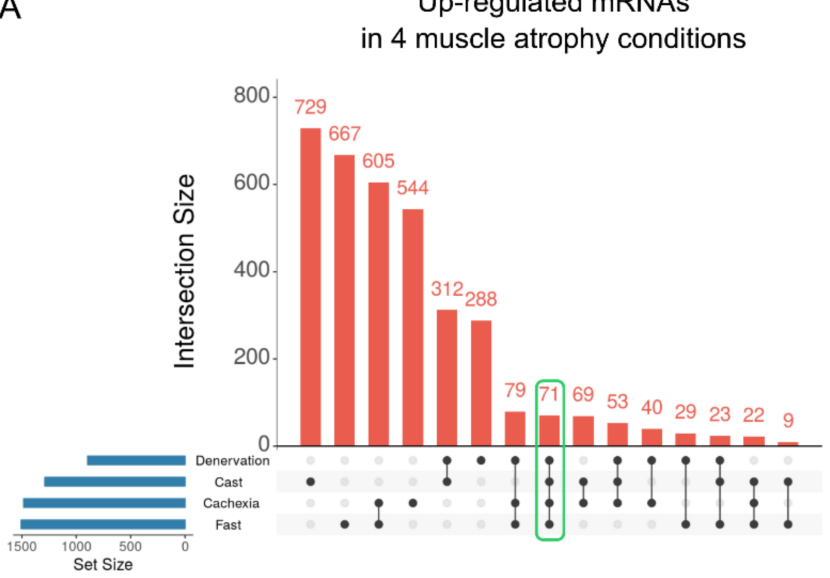

B

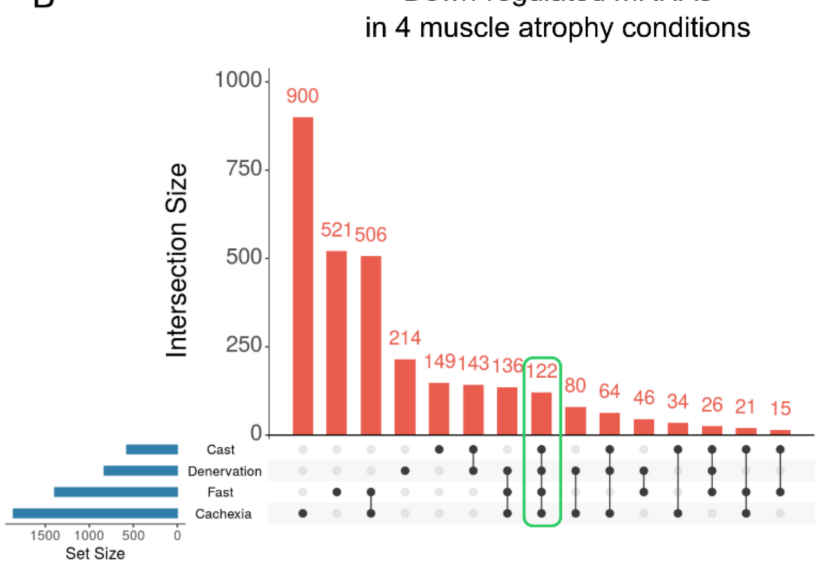

C

Up-regulated mRNAs in 4 muscle atrophy conditions

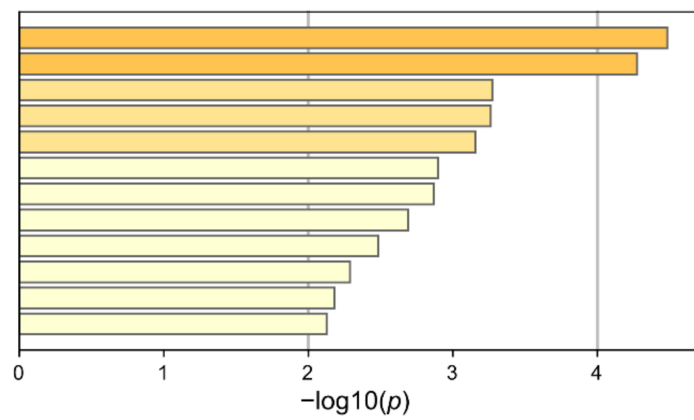

GO:0002695: negative regulation of leukocyte activation GO:0010830: regulation of myotube differentiation GO:0045765: regulation of angiogenesis

GO:1902041: regulation of extrinsic apoptotic signaling pathway via death domain receptors GO:0002062: chondrocyte differentiation

R-MMU-71291: Metabolism of amino acids and derivatives

GO:1901215: negative regulation of neuron death

GO:0006094: gluconeogenesis

R-MMU-6811434: COPI-dependent Golgi-to-ER retrograde traffic

mmu04066: HIF-1 signaling pathway

GO:0071901: negative regulation of protein serine/threonine kinase activity

GO:1902532: negative regulation of intracellular signal transduction

D

Down-regulated mRNAs in 4 muscle atrophy conditions

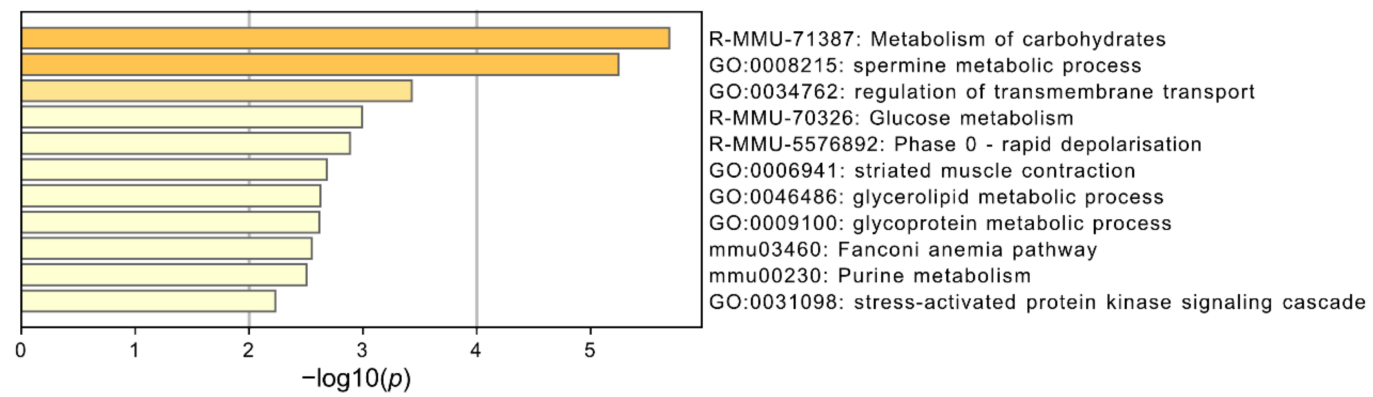

Figure 4. Functional enrichment analysis of mRNAs with altered expression across all four muscle atrophy conditions. The UpSet plot shows the intersections of up- (A) or down-regulated (B) mRNAs among four muscle atrophy conditions. Each column corresponds to an exclusive intersection containing the elements of the set indicated by the black circles but not the elements of any other set. Set sizes related to blue horizontal bars represent the total number of differentially expressed mRNAs for each condition. The intersection sizes between different muscle atrophy conditions represent exclusive intersections (i.e., intersection sets that are not a subset of other intersection sets). The number of mRNAs whose expression was commonly altered in all four muscle atrophy conditions is encircled by the green line. The results of functional enrichment analyses of up- (C) or down-regulated (D) mRNAs common to the four muscle atrophy conditions are shown by a bar graph generated using Metascape. The $x$-axis represents the $-\log 10$ ( $p$-value). 


\subsection{Identification of IncRNAs That Are Altered in Skeletal Muscle Atrophy}

We next extracted lncRNAs that were up- or down-regulated in all four atrophy conditions. Five and 28 annotated lncRNAs were up- and down-regulated in all muscle atrophy conditions, respectively (Figure 5A,B and Table S3). Seven unannotated lncRNAs were commonly up-regulated, and 11 unannotated lncRNAs were commonly down-regulated in all four muscle atrophy conditions, respectively (Figure 5C,D and Table S4).

A

$$
\begin{aligned}
& \text { Up-regulated annotated IncRNAs } \\
& \text { in } 4 \text { muscle atrophy conditions }
\end{aligned}
$$

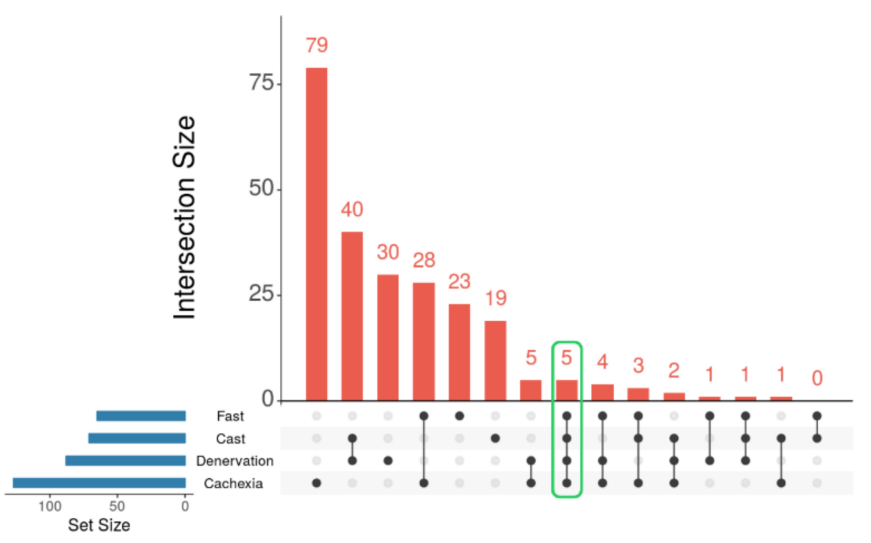

C

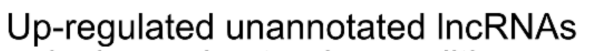

in 4 muscle atrophy conditions

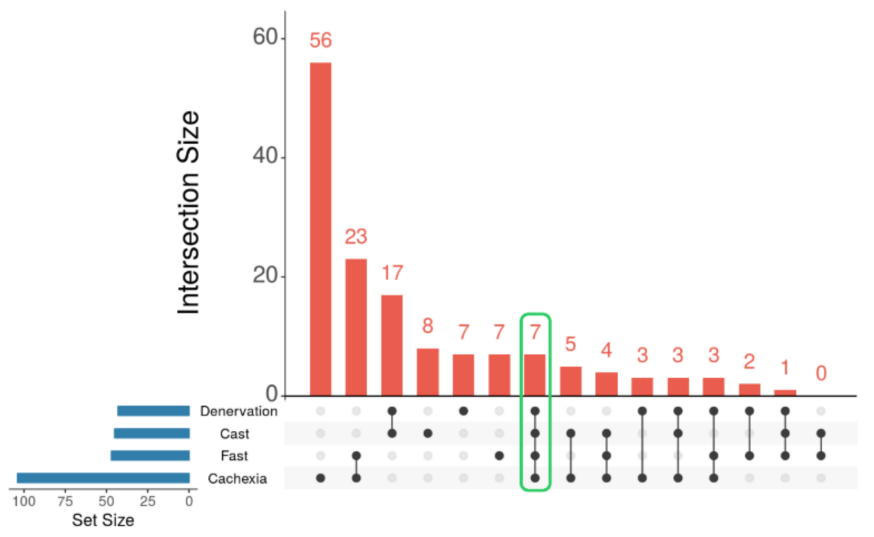

B

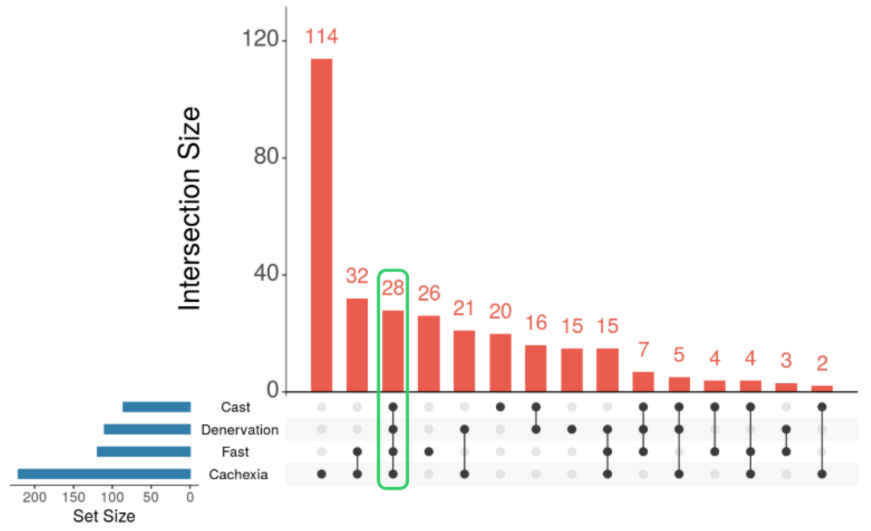

D

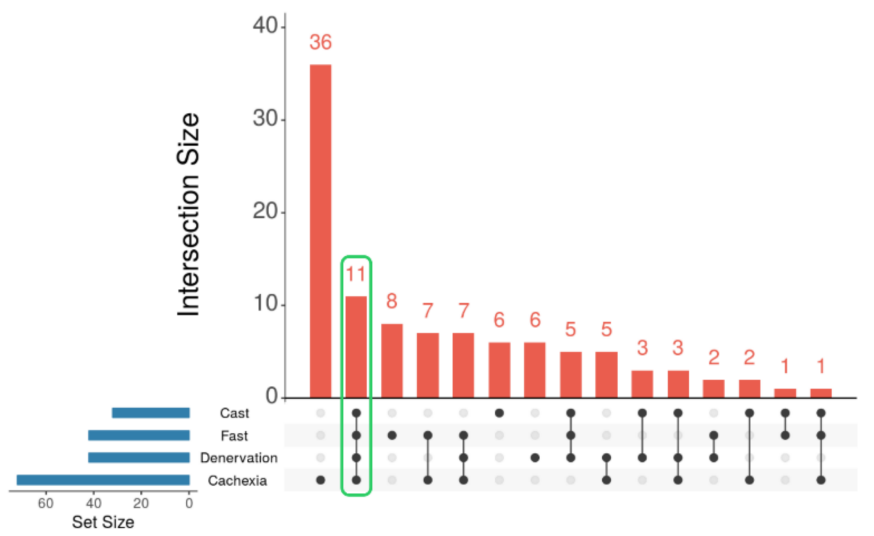

Figure 5. Identification of lncRNAs that are commonly altered in four muscle atrophy conditions. The UpSet plot represents the number of up- (A) or down-regulated (B) annotated lncRNAs by conditions identified by linked dots below the $x$-axis. Set sizes related to blue horizontal bars represent the total number of differentially expressed lncRNAs for each condition. The intersections represent the up- (C) or down-regulated (D) unannotated lncRNAs among muscle atrophy conditions. The number of lncRNAs that were commonly altered in all four muscle atrophy conditions is encircled by the green line.

We estimated the molecular functions of identified lncRNAs by a co-expression network analysis. Correlation analysis based on Spearman's correlation coefficients for the identified $\operatorname{lncRNAs}$ (33 annotated and 18 unannotated lncRNAs) and 193 mRNAs (71 upregulated and 122 down-regulated mRNAs) and following functional enrichment analysis of correlated mRNAs showed that the expression changes of four identified lncRNAs, including down-regulated annotated lncRNAs (linc-Myh (2310065F04Rik) and Gm49794) and down-regulated unannotated lncRNAs (G408 and G10427), were related to changes in mRNAs involved in small molecule biosynthetic processes (Figure S1A). The lncRNA-mRNA co-expression networks also indicated that decreased expression levels of the above lncRNAs affect small molecule biosynthetic processes during muscle atrophy (Figure S1B,C). 
Finally, the results of the RNA-Seq analysis were validated by quantitative PCR (qPCR). We performed a quantitative analysis of multiple identified lncRNAs, including the four lncRNAs extracted from the lncRNA-mRNA network analysis. It should be noted that G408, identified as an unannotated lncRNA in this study, was identical to the lncRNA Gm46085 based on a detailed sequence analysis. Therefore, G408/Gm46085 was regarded as an annotated lncRNA in the qPCR analysis. Six annotated lncRNAs (linc-Myh, Oip5os1, 2310015D24Rik, Gm4544, Gm46085, and Gm49794) were evaluated in each muscle atrophy condition. The quantification results obtained by qPCR were almost identical to the results of the RNA-Seq analysis except for the expression of 2310015D24Rik in the casting model (Figure 6A-F). For unannotated lncRNAs, in addition to G10427, 11 lncRNAs were randomly selected from identified unannotated lncRNAs that were commonly up- or down-regulated in all muscle atrophy conditions. The qPCR results for the up-regulated unannotated lncRNAs were generally consistent with the RNA-Seq results, although some differences were not statistically significant (Figure 7A-D). The expression levels of the eight down-regulated unannotated lncRNAs decreased significantly in all atrophy conditions except for the casting samples (Figure 7E-L). These results suggest that the lncRNAs identified by our RNA-Seq analysis are generally involved in the development or progression of skeletal muscle atrophy.

A

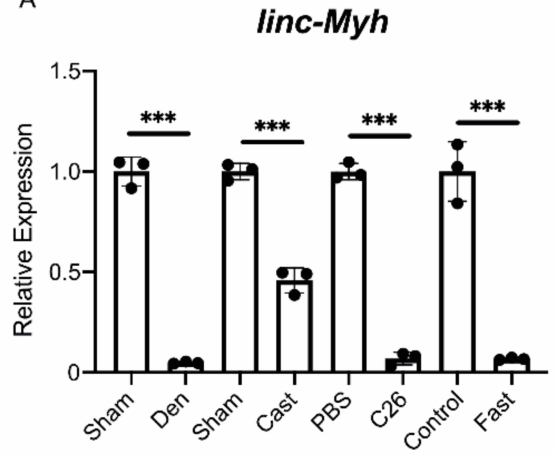

D

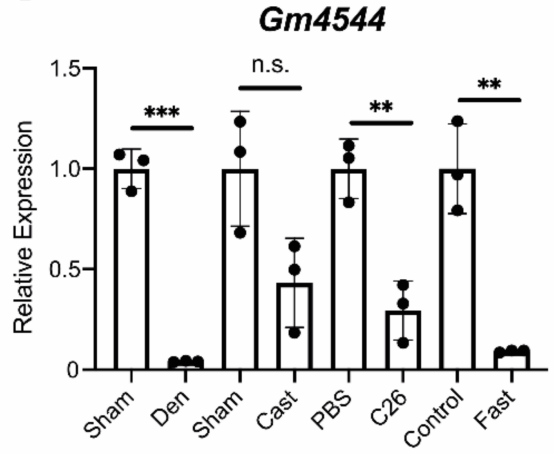

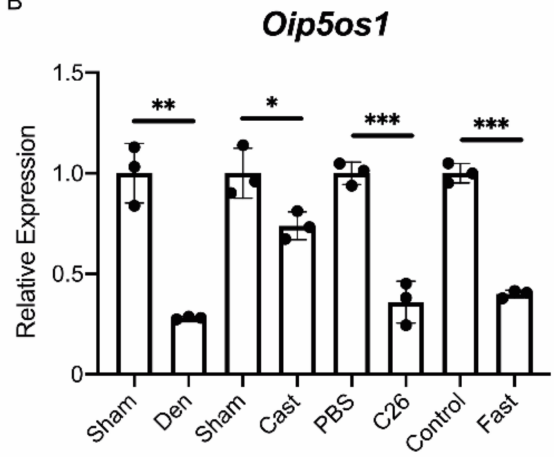

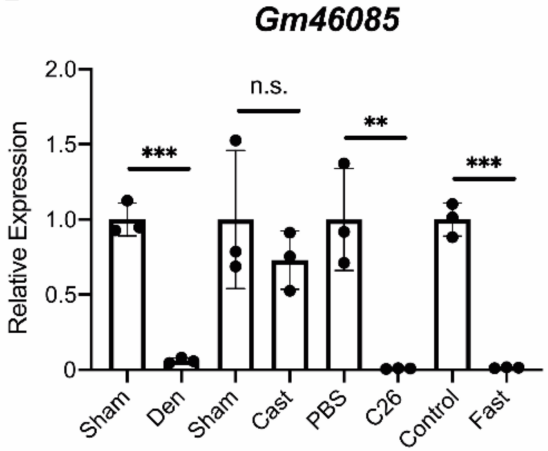

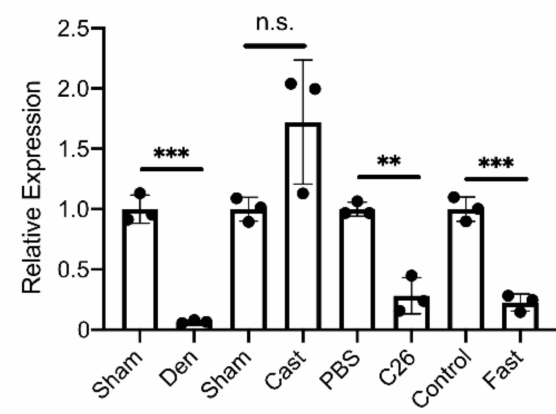

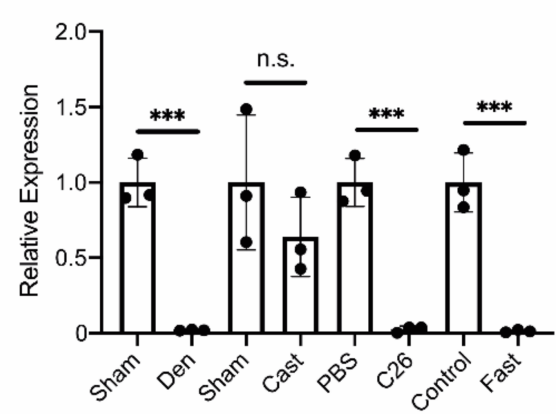

Figure 6. Quantification of six differentially expressed annotated lncRNAs. (A-E) The quantitative RT-PCR (qRT-PCR) results for the expression of linc-Myh (A), Oip5os1 (B), 2310015D24Rik (C), Gm4544 (D), Gm46085 (E), and Gm49794 (F) in four muscle atrophy conditions. Sham; sham-operated tibialis anterior (TA) muscles. Den; denervated TA muscles. Cast; casting-operated TA muscles. PBS; TA muscles of phosphate-buffered saline (PBS)-injected control mice. C26; TA muscles of C26 tumor-bearing mice. Control; TA muscles of mice provided water and food ad libitum. Fast; TA muscles of fasting-treated mice. Data were normalized to Rpl26 expression and are shown as relative expression. $n=3$ biological replicates per group, mean $\pm \mathrm{SD}$. Dots represent the value of each sample. ${ }^{*} p<0.05 ;{ }^{* *} p<0.01 ;{ }^{* * *} p<0.001 ; n$. s.: not significant. 

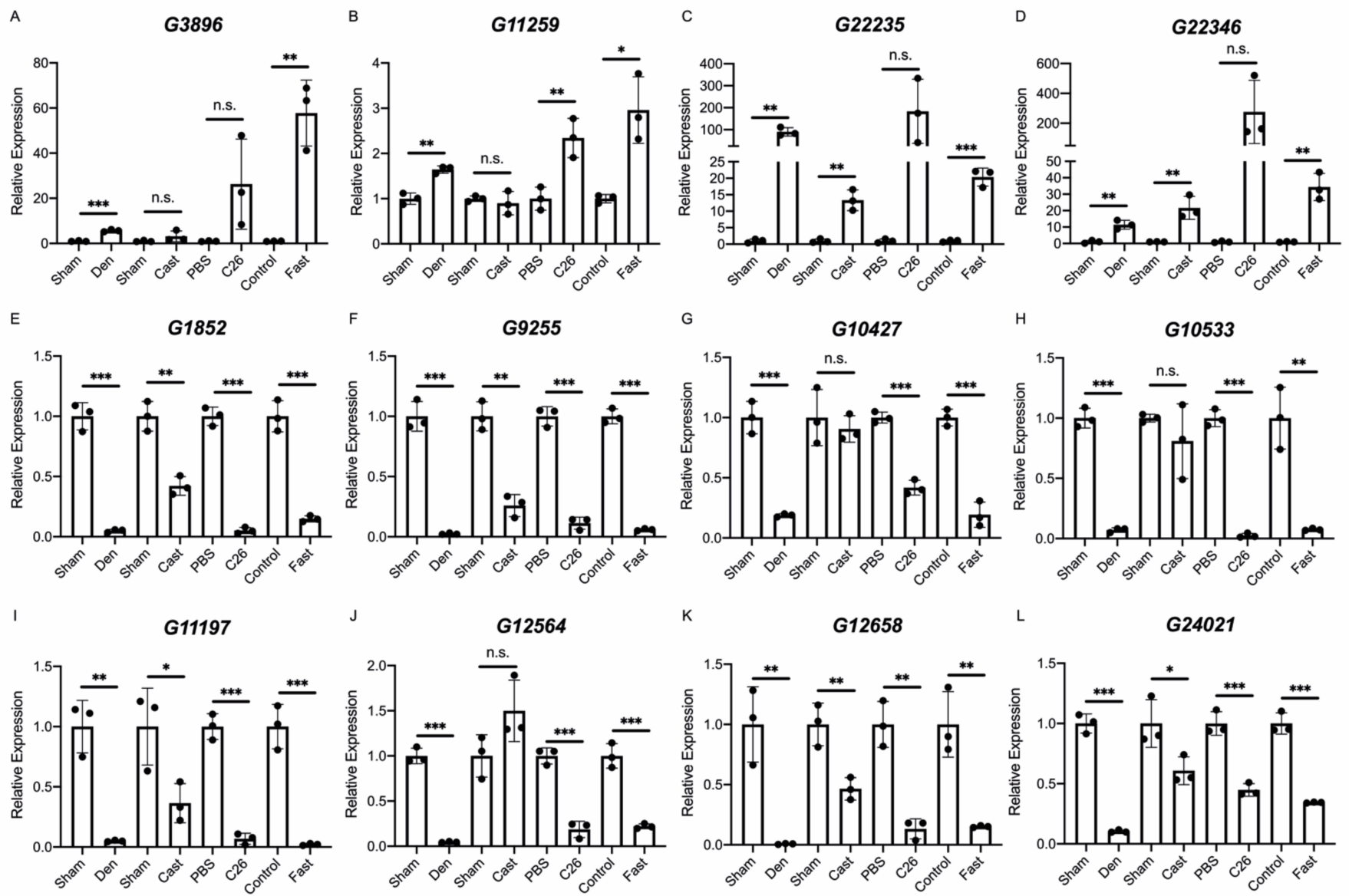

Figure 7. Changes in the expression of unannotated lncRNAs in four muscle atrophy conditions. (A-L) The qRT-PCR results for G3896 (A), G11259 (B), G22235 (C), G22346 (D), G1852 (E), G9255 (F), G10427 (G), G10533 (H), G11197 (I), G12564 (J), G12658 (K), and G24021 (L) in four muscle atrophy conditions. Data were normalized to Rpl26 expression and are shown as relative expression. $n=3$ per group, mean \pm SD. Dots represent the value of each sample. ${ }^{*} p<0.05 ;{ }^{* *} p<0.01 ;{ }^{* *} p<0.001 ; n . s$, not significant.

\section{Discussion}

Recent global transcriptome analyses have identified numerous lncRNAs involved in the regulation of skeletal muscle formation and differentiation, indicating the importance of lncRNAs in myogenesis [24]. However, little is known about the functions of lncRNAs in the regulation of skeletal muscle mass. We have previously examined the expression levels of 12 annotated lncRNAs in six muscle atrophy conditions [23]. Although these lncRNAs were up- or down-regulated in each muscle atrophy condition, none of them showed consistent expression changes across all muscle atrophy conditions. In this study, we performed a transcriptome analysis using four muscle atrophy models (denervation, casting, fasting, and cancer-induced cachexia) and found thousands of IncRNAs that were differentially expressed in each muscle atrophy. One-third of these lncRNAs were unannotated lncRNAs in intergenic regions, indicating that RNA-Seq could be a powerful tool not only for revealing the expression changes of known lncRNAs but also for identifying novel lncRNAs associated with muscle atrophy. Furthermore, we identified 33 annotated and 18 unannotated lncRNAs that showed altered expression across four muscle atrophy conditions. A quantitative PCR analysis of $18 \mathrm{lncRNAs}$ randomly chosen from the total set of 51 lncRNAs confirmed the RNA-Seq results. Taken together, we identified 51 lncRNAs associated with muscle atrophy, providing candidates for further functional analyses to elucidate the molecular mechanisms underlying the development or progression of skeletal muscle atrophy. 
Molecular functions for most of the lncRNAs identified in this study are still unknown. However, the expression levels of Oip5os1 and linc-Myh were substantially decreased in all muscle atrophy conditions. Oip5os1, also known as OIP5-AS1/cyrano, was initially implicated in the development of the brain and eye by morpholino studies in zebrafish [40]. However, subsequent studies have shown that Oip5os1 mutant mice and zebrafish exhibited normal development [41]. More recently, Yang et al. found that Oip5os1 contributed to myogenesis [42]. After myogenic differentiation, increased Oip5os1 expression increased the production of Mef2c protein, a myogenic transcription factor, by recruiting HuR to the $3^{\prime}$ utr of Mef2c mRNA [42]. We observed that Mef2c mRNA levels decreased significantly after fasting and cachexia (Table S1). Mef2 was positively related to muscle mass in adult skeletal muscle [43]; therefore, Oip5os1 may be involved in the regulation of muscle mass via Mef2 in muscle atrophy conditions. Linc-Myh is located in the Myh3-Myh13 gene cluster, and a previous study has shown that the knockdown of linc-Myh increased the expression levels of slow skeletal muscle fiber genes, suggesting that linc-Myh is involved in muscle fiber-type specification in mice [44]. However, Schutt et al. recently generated linc-Myh-knockout mice and found no change in the muscle fiber- type distribution and instead observed muscle hypertrophy [45]. Considering the fact that linc-Myh restricts the proliferation of satellite cells (adults muscle stem cells) via the INO80 chromatin remodeling complex [45], an increased number of satellite cells would induce muscle hypertrophy in linc-Myh-knockout mice. Further analyses will reveal the precise role of the decreased expression of linc-Myh in muscle atrophy. Intriguingly, in our co-expression network analysis of lncRNAs and mRNAs, we identified four lncRNAs, linc-Myh, Gm48065, Gm49794, and G10427, that were involved in small molecule biosynthetic processes. Although the exact role of this pathway in the regulation of skeletal muscle mass remains unclear, it is noteworthy that Slc39a14, $M t 1$, and $M t 2$ in this pathway are associated with muscle mass [46,47]. Slc39a14 (Zip14) encodes a zinc transporter, and its expression in skeletal muscle tissues is highly activated in response to lipopolysaccharide-induced inflammation. In mice, Slc39a14 knockout leads to muscle atrophy and metabolic endotoxemia, indicating that this gene is required to maintain skeletal muscle mass [46]. However, knockout mice of both $M t 1$ and $M t 2$, which encode metallothioneins, show muscle hypertrophy with increased muscle strength via the activation of the IGF-1/Akt/mTOR pathway [47]. Therefore, lncRNAs are associated with the development and progression of muscle atrophy via the regulation of small molecule biosynthetic processes.

Skeletal muscle atrophy is the main physiological change common to all four models used in this study. However, the four models also show differences in muscle fiber-type, stem cell activity, and mitochondrial function. For example, denervation and casting induce a slow-to-fast fiber-type shift, whereas cancer cachexia and fasting cause a fast-to-slow fiber-type shift [48]. In addition, denervation and cancer cachexia increase the activity of satellite cells [49,50], and casting and fasting decrease the number of satellite cells [51,52]. Recently, Li et al. found that the lncRNA MSTRG.42019 was associated with skeletal muscle fiber-type switching in pigs [53]. Several lncRNAs, such as LncMyoD, SAM, H19, and CTTNIT1, affect the activity of satellite cells in mice, pigs, and sheep [54-57]. The in vivo silencing of Pvt1 results in increases in the size and number of mitochondria [20]. However, the identification of additional novel lncRNAs is needed to elucidate the physiological roles of lncRNAs in fiber-type shifting, satellite cell activity, and mitochondrial function in skeletal muscle. Our data, obtained by optimizing the comparisons (for example, comparing denervation and casting treatments with cancer cachexia and fasting treatments to identify lncRNAs associated with fiber-type switching), not only provide candidate therapeutic targets for human muscle atrophy but also provide insights into the roles of lncRNAs in skeletal muscle physiology. 


\section{Materials and Methods}

\subsection{Animal Experiments}

Mice (C57BL/6J and CD2F1) were purchased from the Japan SLC and Charles River Laboratories Japan. Mice were housed in cages with a constant temperature $\left(24^{\circ} \mathrm{C}\right)$ and a $12 \mathrm{~h}$ light: $12 \mathrm{~h}$ dark cycle and were provided water and food ad libitum. During the fasting experiments, no food intake was allowed. C57BL/6J mice were used for denervation, atrophy by casting and fasting, and CD2F1 mice were used for cachexia treatment. The detailed protocols for each muscle atrophy experiment and the changes in the skeletal muscle weight were described previously [23].

\subsection{RNA-Seq Library Construction, Sequencing, and Data Analysis}

RNA-Seq libraries were prepared as described previously [22]. In brief, after the induction of muscle atrophy, total RNA isolated from the tibialis anterior muscles was purified using the miRNeasy Mini Kit (QIAGEN, Hilden, Germany) with DNase I (QIAGEN, Hilden, Germany), according to the manufacturer's protocol. One microgram of total RNA was used for the purification of Poly(A)+ RNAs using the NEBNext Poly(A) mRNA Magnetic Isolation Module (New England Biolabs, Ipswich, MA, USA). The RNA-Seq libraries were constructed using the NEBNext Ultra RNA Library Prep Kit for Illumina (New England Biolabs, Ipswich, MA, USA), according to the manufacturer's protocol. The libraries were sequenced to obtain 124-bp single-end reads for each sample (two biological replicates per sample) using an Illumina HiSeq 1500. bcl2fastq 1.8.4 was used for base-calling. Raw sequence data were deposited into the DNA Data Bank of Japan (DDBJ; Accession IDs DRR238781 to DRR238792, Table S5). To analyze the expression profiles of mRNAs and lncRNAs in cachexia samples, published RNA-Seq data (GSE65936) [58] were used.

Raw sequence data were quality-trimmed using FastQC ver. 0.11 .3 (https:/ / www. bioinformatics.babraham.ac.uk/projects/fastqc/ 26 February 2021) with the command "-Q 33 -t 20130 ." The reads were aligned to the GENCODE GRCm38_p6_genome using Hisat2 v. 2.2.0 [59] with default parameters. Mapped reads were converted to Bam files using SAMtools v. 1.3.1 [60]. Read counts were obtained using StringTie v. 2.1.3b [59] and taco v. 0.7.3 [61] from the Bam files using the gencode_vM25_Annotation.gtf file with default parameters. Gene_count_matrix data produced by read counting were used for statistical analyses of differentially expressed genes by DESeq2 v. 1.26.0 [62] with the Wald test (cut-offs: base-mean $>10$, false discovery rate (adjusted $p$-value, padj) $<0.05$, and log2 fold change of $>1$ or $<-1$ ).

The protein-coding potential was calculated by the Coding Potential Assessment Tool (CPAT) [63]. In this study, lncRNAs were defined as RNAs categorized as lncRNAs by CPAT with no CPAT coding label. mRNAs and lncRNAs were counted on the basis of the gene_id. The transcript_id was used to examine the chromosomal location, number of exons, RNA length, and ORF size. An enrichment analysis was performed using Metascape [64]. The lncRNA-mRNA network was analyzed by calculating correlation coefficients from normalized read count data for lncRNAs and mRNAs whose expression levels were altered in all muscle atrophy conditions. According to a previous report [65], Spearman's correlation coefficients $>0.9$ (positive) or $<-0.9$ (negative) and a value of $p<0.05$ were considered significant. After an enrichment analysis of extracted mRNAs by Metascape, the co-expression network between lncRNAs and mRNAs related to small molecule biosynthetic processes was visualized by Cytoscape [66].

\subsection{RNA Purification, Reverse Transcription, and $q P C R$}

One microgram of total RNA was used to generate cDNAs by reverse transcription using Protoscript II Reverse Transcriptase (New England Biolabs, Ipswich, MA, USA) with random primers. Quantitative PCR was performed using SYBR Premix Ex Taq (Takara, Kusatsu, Japan). Relative gene expression was determined using a $\Delta \Delta \mathrm{Ct}$ method and normalized to a reference gene (Rpl26 expression). Values are shown as each control (sham- 
operated muscles, phosphate-buffered saline (PBS)-injected control mice, or muscles of mice provided water and food ad libitum) is 1 . The primers are listed in Table 1. Statistical analyses were performed using unpaired, two-tailed Student's $t$-tests. A value of $p<0.05$ was considered statistically significant.

Table 1. Primer sequences used for quantitative PCR.

\begin{tabular}{ccc}
\hline Target Name & Forward & Reverse \\
\hline linc-Myh & GTGCAGCCAGAACAAGACAG & CAAGATGGGAGGCTCTCAAA \\
Oip5os1 & ATAAACAGGCGCCACCATCA & CAGCACAGCCTGAGTCTGAA \\
$2310015 D 24$ Rik & AGCTATCCACAGCCAGAGGA & GACGTCAAAGGTCTGCAGGA \\
Gm4544 & CTGACTCCCCCAAGTTGTCC & GAGCTGTGATTGCAGATGCG \\
Gm46085 & AAACCCCCAAAACCCCAACT & CCCTGGGTCCTCATTTTCCC \\
Gm49794 & GTCAACTGCCTTAGCCAGGT & TCACAGCTTCTGCACCTCAG \\
G3896 & AGAGGAGGCAGGGTAACGAT & CCGTGGGTTCTGCTTTTTGG \\
G11259 & AAACCGTACCACTGGAGCAG & CAGTTTCCCTATGCAGCCCA \\
G22235 & TTTTCCCAGTGCCCAACAGT & ACACTGAATACCCTGGCTGC \\
G22346 & TTTCTGTGACTCCGTGACCG & TTGCAAGGAGATGGCGTTCT \\
G1852 & TTGCCATCACCAGTAGCCTG & GGGAGTGGCTCTCTCAGAGA \\
G9255 & AATCCTCTCCCCAGAGCTGT & AGGAGGACCAATACCCAGGG \\
G10427 & CCACCTTTGACTCAGGCCTT & TATGATGAGCTGGGACCCCA \\
G10533 & TCGAGTCTGTTCCACATGGC & TCCTCAGGGCTAGGACTTCC \\
G11197 & AAGAGAGTGTTTCCTGGCCG & TGAGGGATTGCATGTGCCAT \\
G12564 & GCCTGAGGACATTGTGGTGA & TGTCTTCTTCAGGCAGCCAG \\
G12658 & GCAGAGACCTTTCTGACCCC & GGTGGTGACAGAGAAGGGTG \\
G24021 & GTTCCTGTACCTGGGGTTGG & ACATGTGGGTGAGCTGATGG \\
Rpl26 & GGTCTATGCCCATTCGGAAGG & TCGTTCGATGTAGATGACGTACT \\
\hline
\end{tabular}

Supplementary Materials: The following are available online at https:/ / www.mdpi.com/1422-0 067/22/5/2558/s1, Figure S1: Co-expression network for lncRNAs and mRNAs whose expression levels were altered in all atrophy conditions, Table S1: Up- or down-regulated mRNAs and lncRNAs in each muscle atrophy condition, Table S2: Up- or down-regulated mRNAs across four muscle atrophy conditions, Table S3: Up- or down-regulated annotated lncRNAs across four muscle atrophy conditions, Table S4: Up- or down-regulated unannotated lncRNAs across four muscle atrophy conditions, Table S5: List of raw sequence data.

Author Contributions: Conceptualization, K.H.; methodology, K.H.; validation, K.H.; formal analysis, K.H.; investigation, K.H., M.N., Y.K., H.I., and H.K.; resources, K.H., M.N., and K.T.; data curation, K.H.; writing—original draft preparation, K.H.; writing—review and editing, K.H., M.N., Y.K., H.I., H.K., and K.T.; visualization, K.H. and K.T.; supervision, K.H. and K.T.; project administration, K.H. and K.T.; funding acquisition, K.H. and K.T. All authors have read and agreed to the published version of the manuscript.

Funding: This work was supported in part by JSPS KAKENHI (19H03427 and 20K07315), Intramural Research Grants (29-4 and 2-5) for Neurological and Psychiatric Disorders of NCNP, and a Grant-inAid from the Mochida Memorial Foundation for Medical and Pharmaceutical Research.

Institutional Review Board Statement: Animal experiments were approved by the Institutional Animal Care and Use Committee of Fujita Health University, Japan. All animal experiments were performed under the ethical standards laid down in the 1964 Declaration of Helsinki and its later amendments.

Data Availability Statement: The RNA-seq raw data for each sample reported in this study were deposited into the DDBJ Sequence Read Archive under the accession No. DRA010545.

Conflicts of Interest: The authors declare no conflict of interest. The funders had no role in the design of the study; in the collection, analyses, or interpretation of data; in the writing of the manuscript; or in the decision to publish the results. 


\section{References}

1. Hon, C.-C.; Ramilowski, J.A.; Harshbarger, J.; Bertin, N.; Rackham, O.J.L.; Gough, J.; Denisenko, E.; Schmeier, S.; Poulsen, T.M.; Severin, J.; et al. An atlas of human long non-coding RNAs with accurate $5^{\prime}$ ends. Nature 2017, 543, 199-204. [CrossRef]

2. Ma, L.; Cao, J.; Liu, L.; Du, Q.; Li, Z.; Zou, D.; Bajic, V.B.; Zhang, Z. LncBook: A curated knowledgebase of human long non-coding RNAs. Nucleic Acids Res. 2019, 47, D128-D134. [CrossRef] [PubMed]

3. Ali, T.; Grote, P. Beyond the RNA-dependent function of LncRNA genes. eLife 2020, 9, e60583. [CrossRef]

4. Kozomara, A.; Birgaoanu, M.; Griffiths-Jones, S. miRBase: From microRNA sequences to function. Nucleic Acids Res. 2019, 47, D155-D162. [CrossRef]

5. Bao, Z.; Yang, Z.; Huang, Z.; Zhou, Y.; Cui, Q.; Dong, D. LncRNADisease 2.0: An updated database of long non-coding RNA-associated diseases. Nucleic Acids Res. 2019, 47, D1034-D1037. [CrossRef]

6. Zhao, H.; Shi, J.; Zhang, Y.; Xie, A.; Yu, L.; Zhang, C.; Lei, J.; Xu, H.; Leng, Z.; Li, T.; et al. LncTarD: A manually-curated database of experimentally-supported functional lncRNA-target regulations in human diseases. Nucleic Acids Res. 2019, 48, D118-D126. [CrossRef]

7. Aznaourova, M.; Schmerer, N.; Schmeck, B.; Schulte, L.N. Disease-Causing Mutations and Rearrangements in Long Non-coding RNA Gene Loci. Front. Genet. 2020, 11, 527484. [CrossRef] [PubMed]

8. Larsson, L.; Degens, H.; Li, M.; Salviati, L.; Lee, Y.I.; Thompson, W.; Kirkland, J.L.; Sandri, M. Sarcopenia: Aging-Related Loss of Muscle Mass and Function. Physiol. Rev. 2019, 99, 427-511. [CrossRef] [PubMed]

9. Gao, Y.; Arfat, Y.; Wang, H.; Goswami, N. Muscle atrophy induced by mechanical unloading: Mechanisms and potential coun-termeasures. Front. Physiol. 2018, 9, 235. [CrossRef]

10. Picot, J.; Hartwell, D.; Harris, P.; Mendes, D.; Clegg, A.J.; Takeda, A. The effectiveness of interventions to treat severe acute malnutrition in young children: A systematic review. Health Technol. Assess. 2012, 16, 1-316. [CrossRef]

11. Rohm, M.; Zeigerer, A.; Machado, J.; Herzig, S. Energy metabolism in cachexia. EMBO Rep. 2019, 20, e47258. [CrossRef] [PubMed]

12. Suzuki, T.; Palus, S.; Springer, J. Skeletal muscle wasting in chronic heart failure. ESC Heart Fail. 2018, 5, 1099-1107. [CrossRef]

13. Casas, C.; Manzano, R.; Vaz, R.; Osta, R.; Brites, D. Synaptic Failure: Focus in an Integrative View of ALS. Brain Plast. 2016, 1, 159-175. [CrossRef] [PubMed]

14. Schiaffino, S.; Dyar, K.A.; Ciciliot, S.; Blaauw, B.; Sandri, M. Mechanisms regulating skeletal muscle growth and atrophy. FEBS J. 2013, 280, 4294-4314. [CrossRef] [PubMed]

15. Rodriguez, J.; Vernus, B.; Chelh, I.; Cassar-Malek, I.; Gabillard, J.-C.; Sassi, A.H.; Seiliez, I.; Picard, B.; Bonnieu, A. Myostatin and the skeletal muscle atrophy and hypertrophy signaling pathways. Cell. Mol. Life Sci. 2014, 71, 4361-4371. [CrossRef] [PubMed]

16. Braun, T.P.; Marks, D.L. The regulation of muscle mass by endogenous glucocorticoids. Front. Physiol. 2015, 6, 12. [CrossRef]

17. Sartori, R.; Romanello, V.; Sandri, M. Mechanisms of muscle atrophy and hypertrophy: Implications in health and disease. Nat. Commun. 2021, 12, 330. [CrossRef]

18. Hitachi, K.; Tsuchida, K. Role of microRNAs in skeletal muscle hypertrophy. Front. Physiol. 2014, 4, 408. [CrossRef]

19. Sun, L.; Si, M.; Liu, X.; Choi, J.M.; Wang, Y.; Thomas, S.S.; Peng, H.; Hu, Z. Long-noncoding RNA Atrolnc-1 promotes muscle wasting in mice with chronic kidney disease. J. Cachex. Sarcopenia Muscle 2018, 9, 962-974. [CrossRef]

20. Alessio, E.; Buson, L.; Chemello, F.; Peggion, C.; Grespi, F.; Martini, P.; Massimino, M.L.; Pacchioni, B.; Millino, C.; Romualdi, C.; et al. Single cell analysis reveals the involvement of the long non-coding RNA Pvt1 in the modulation of muscle atrophy and mi-tochondrial network. Nucleic Acids Res. 2019, 47, 1653-1670. [CrossRef]

21. Hitachi, K.; Nakatani, M.; Takasaki, A.; Ouchi, Y.; Uezumi, A.; Ageta, H.; Inagaki, H.; Kurahashi, H.; Tsuchida, K. Myogenin promoter-associated lncRNA Myoparr is essential for myogenic differentiation. EMBO Rep. 2019, 20, e47468. [CrossRef]

22. Hitachi, K.; Nakatani, M.; Tsuchida, K. Long Non-Coding RNA Myoparr Regulates GDF5 Expression in Denervated Mouse Skeletal Muscle. Non-Coding RNA 2019, 5, 33. [CrossRef]

23. Hitachi, K.; Nakatani, M.; Funasaki, S.; Hijikata, I.; Maekawa, M.; Honda, M.; Tsuchida, K. Expression Levels of Long Non-Coding RNAs Change in Models of Altered Muscle Activity and Muscle Mass. Int. J. Mol. Sci. 2020, 21, 1628. [CrossRef]

24. Hitachi, K.; Tsuchida, K. The chemical biology of long noncoding RNAs. RNA Technol. 2020, 11, 431-463. [CrossRef]

25. Fox, D.K.; Ebert, S.M.; Bongers, K.S.; Dyle, M.C.; Bullard, S.A.; Dierdorff, J.M.; Kunkel, S.D.; Adams, C.M. p53 and ATF4 mediate distinct and additive pathways to skeletal muscle atrophy during limb immobilization. Am. J. Physiol. Metab. 2014, 307, E245-E261. [CrossRef] [PubMed]

26. Tsujinaka, T.; Fujita, J.; Ebisui, C.; Yano, M.; Kominami, E.; Suzuki, K.; Tanaka, K.; Katsume, A.; Ohsugi, Y.; Shiozaki, H.; et al. Interleukin 6 receptor antibody inhibits muscle atrophy and modulates proteolytic systems in interleukin 6 transgenic mice. J. Clin. Investig. 1996, 97, 244-249. [CrossRef]

27. Mele, A.; Camerino, G.M.; Calzolaro, S.; Cannone, M.; Conte, D.; Tricarico, M. Dual response of the KATP channels to staurosporine: A novel role of SUR2B, SUR1 and Kir6.2 subunits in the regulation of the atrophy in different skeletal muscle phenotypes. Biochem. Pharmacol. 2014, 91, 266-275. [CrossRef]

28. Barash, I.A.; Mathew, L.; Lahey, M.; Greaser, M.L.; Lieber, R.L. Muscle LIM protein plays both structural and functional roles in skeletal muscle. Am. J. Physiol. Physiol. 2005, 289, C1312-C1320. [CrossRef] [PubMed]

29. Cui, C.; Han, S.; Tang, S.; He, H.; Shen, X.; Zhao, J.; Chen, Y.; Wei, Y.; Wang, Y.; Zhu, Q.; et al. The Autophagy Regulatory Molecule CSRP3 Interacts with LC3 and Protects Against Muscular Dystrophy. Int. J. Mol. Sci. 2020, 21, 749. [CrossRef] [PubMed] 
30. Kaya-Çopur, A.; Marchiano, F.; Hein, M.Y.; Alpern, D.; Russeil, J.; Luis, N.M.; Mann, M.; Deplancke, B.; Habermann, B.H.; Schnorrer, F. The Hippo pathway controls myofibril assembly and muscle fiber growth by regulating sarcomeric gene expression. eLife 2021, 10, e63726. [CrossRef]

31. MacPherson, P.C.D.; Farshi, P.; Goldman, D. Dach2-Hdac9 signaling regulates reinnervation of muscle endplates. Development 2015, 142, 4038-4048. [CrossRef] [PubMed]

32. Nagahara, Y.; Shimazawa, M.; Tanaka, H.; Ono, Y.; Noda, Y.; Ohuchi, K.; Tsuruma, K.; Katsuno, M.; Sobue, G.; Hara, H. Glycoprotein nonmetastatic melanoma protein B ameliorates skeletal muscle lesions in a SOD1G93A mouse model of amyotrophic lateral sclerosis. J. Neurosci. Res. 2015, 93, 1552-1566. [CrossRef]

33. Costamagna, D.; Duelen, R.; Penna, F.; Neumann, D.; Costelli, P.; Sampaolesi, M. Interleukin-4 administration improves muscle function, adult myogenesis, and lifespan of colon carcinoma-bearing mice. J. Cachex. Sarcopenia Muscle 2020, 11, 783-801. [CrossRef]

34. Wang, X.; Blagden, C.; Fan, J.; Nowak, S.J.; Taniuchi, I.; Littman, D.R.; Burden, S.J. Runx1 prevents wasting, myofibrillar dis-organization, and autophagy of skeletal muscle. Gene Dev. 2005, 19, 1715-1722. [CrossRef]

35. Ng, D.C.; Carlsen, R.C.; Walsh, N.A. Neural regulation of the formation of skeletal muscle phosphorylase kinase holoenzyme in adult and developing rat muscle. Biochem. J. 1997, 325, 793-800. [CrossRef] [PubMed]

36. Chao, L.C.; Zhang, Z.; Pei, L.; Saito, T.; Tontonoz, P.; Pilch, P.F. Nur77 Coordinately Regulates Expression of Genes Linked to Glucose Metabolism in Skeletal Muscle. Mol. Endocrinol. 2007, 21, 2152-2163. [CrossRef]

37. Bongers, K.S.; Fox, D.K.; Kunkel, S.D.; Stebounova, L.V.; Murry, D.J.; Pufall, M.A.; Ebert, S.M.; Dyle, M.C.; Bullard, S.A.; Dierdorff, J.M.; et al. Spermine oxidase maintains basal skeletal muscle gene expression and fiber size and is strongly repressed by conditions that cause skeletal muscle atrophy. Am. J. Physiol. Metab. 2015, 308, E144-E158. [CrossRef] [PubMed]

38. Bonetto, A.; Aydogdu, T.; Kunzevitzky, N.; Guttridge, D.C.; Khuri, S.; Koniaris, L.G.; Zimmers, T.A. STAT3 Activation in Skeletal Muscle Links Muscle Wasting and the Acute Phase Response in Cancer Cachexia. PLoS ONE 2011, 6, e22538. [CrossRef] [PubMed]

39. Stangenberg, L.; Burzyn, D.; Binstadt, B.A.; Weissleder, R.; Mahmood, U.; Benoist, C.; Mathis, D. Denervation protects limbs from inflammatory arthritis via an impact on the microvasculature. Proc. Natl. Acad. Sci. USA 2014, 111, 11419-11424. [CrossRef]

40. Ulitsky, I.; Shkumatava, A.; Jan, C.H.; Sive, H.; Bartel, D.P. Conserved function of lincRNAs in vertebrate embryonic devel-opment despite rapid sequence evolution. Cell 2011, 147, 1537-1550. [CrossRef]

41. Goudarzi, M.; Berg, K.; Pieper, L.M.; Schier, A.F. Individual long non-coding RNAs have no overt functions in zebrafish em-bryogenesis, viability and fertility. eLife 2019, 8, e40815. [CrossRef]

42. Yang, J.-H.; Chang, M.-W.; Pandey, P.R.; Tsitsipatis, D.; Yang, X.; Martindale, J.L.; Munk, R.; De, S.; Abdelmohsen, K.; Gorospe, M. Interaction of OIP5-AS1 with MEF2C mRNA promotes myogenic gene expression. Nucleic Acids Res. 2020, 48, 12943-12956. [CrossRef]

43. Moretti, I.; Ciciliot, S.; Dyar, K.A.; Abraham, R.; Murgia, M.; Agatea, L.; Akimoto, T.; Bicciato, S.; Forcato, M.; Pierre, P.; et al. MRF4 negatively regulates adult skeletal muscle growth by repressing MEF2 activity. Nat. Commun. 2016, 7, 12397. [CrossRef]

44. Sakakibara, I.; Santolini, M.; Ferry, A.; Hakim, V.; Maire, P. Six Homeoproteins and a linc-RNA at the Fast MYH Locus Lock Fast Myofiber Terminal Phenotype. PLoS Genet. 2014, 10, e1004386. [CrossRef]

45. Schutt, C.; Hallmann, A.; Hachim, S.; Klockner, I.; Valussi, M.; Atzberger, A.; Graumann, J.; Braun, T.; Boettger, T. Linc-MYH configures INO 80 to regulate muscle stem cell numbers and skeletal muscle hypertrophy. EMBO J. 2020, 39, e105098. [CrossRef] [PubMed]

46. Kim, J.; Aydemir, T.B.; Jimenez-Rondan, F.R.; Ruggiero, C.H.; Kim, M.-H.; Cousins, R.J. Deletion of metal transporter Zip14 (Slc39a14) produces skeletal muscle wasting, endotoxemia, Mef2c activation and induction of miR-675 and Hspb. Sci. Rep. 2020, 10, 4050. [CrossRef] [PubMed]

47. Summermatter, S.; Bouzan, A.; Pierrel, E.; Melly, S.; Stauffer, D.; Gutzwiller, S.; Nolin, E.; Dornelas, C.; Fryer, C.; LeightonDavies, J.; et al. Blockade of Metallothioneins 1 and 2 Increases Skeletal Muscle Mass and Strength. Mol. Cell. Biol. 2017, 37, e00305-16. [CrossRef]

48. Ciciliot, S.; Rossi, A.C.; Dyar, K.A.; Blaauw, B.; Schiaffino, S. Muscle type and fiber type specificity in muscle wasting. Int. J. Biochem. Cell Biol. 2013, 45, 2191-2199. [CrossRef] [PubMed]

49. Xing, H.; Zhou, M.; Assinck, P.; Liu, N. Electrical stimulation influences satellite cell differentiation after sciatic nerve crush injury in rats. Muscle Nerve 2015, 51, 400-411. [CrossRef]

50. He, W.A.; Berardi, E.; Cardillo, V.M.; Acharyya, S.; Aulino, P.; Thomas-Ahner, J.; Wang, J.; Bloomston, M.; Muscarella, P.; Nau, P.; et al. NF-KB-mediated Pax7 dysregulation in the muscle microenvironment promotes cancer cachexia. J. Clin. Investig. 2013, 123, 4821-4835. [CrossRef]

51. Guitart, M.; Lloreta, J.; Mañas-Garcia, L.; Barreiro, E. Muscle regeneration potential and satellite cell activation profile during recovery following hindlimb immobilization in mice. J. Cell. Physiol. 2018, 233, 4360-4372. [CrossRef] [PubMed]

52. Fauconneau, B.; Paboeuf, G. Effect of fasting and refeeding on in vitro muscle cell proliferation in rainbow trout (Oncorhynchus mykiss). Cell Tissue Res. 2000, 301, 459-463. [CrossRef] [PubMed]

53. Li, R.; Li, B.; Jiang, A.; Cao, Y.; Hou, L.; Zhang, Z.; Zhang, X.; Liu, H.; Kim, K.-H.; Wu, W. Exploring the lncRNAs Related to Skeletal Muscle Fiber Types and Meat Quality Traits in Pigs. Genes 2020, 11, 883. [CrossRef] [PubMed] 
54. Dong, A.; Preusch, C.B.; So, W.-K.; Lin, K.; Luan, S.; Yi, R.; Wong, J.W.; Wu, Z.; Cheung, T.H. A long noncoding RNA, LncMyoD, modulates chromatin accessibility to regulate muscle stem cell myogenic lineage progression. Proc. Natl. Acad. Sci. USA 2020, 117, 32464-32475. [CrossRef] [PubMed]

55. Li, Y.; Yuan, J.; Chen, F.; Zhang, S.; Zhao, Y.; Chen, X.; Lu, L.; Zhou, L.; Chu, C.Y.; Sun, H.; et al. Long noncoding RNA SAM promotes myoblast proliferation through stabilizing Sugt1 and facilitating kinetochore assembly. Nat. Commun. 2020, 11, 2725. [CrossRef]

56. Li, J.; Zhao, W.; Li, Q.; Huang, Z.; Shi, G.; Li, C. Long Non-Coding RNA H19 Promotes Porcine Satellite Cell Differentiation by Interacting with TDP43. Genes 2020,11, 259. [CrossRef]

57. Wu, T.; Wang, S.; Wang, L.; Zhang, W.; Chen, W.; Lv, X.; Li, Y.; Hussain, Z.; Sun, W. Long Noncoding RNA (lncRNA) CTTN-IT1 Elevates Skeletal Muscle Satellite Cell Proliferation and Differentiation by Acting as ceRNA for YAP1 Through Absorbing miR-29a in Hu Sheep. Front. Genet. 2020, 11, 843. [CrossRef] [PubMed]

58. Tseng, Y.-C.; Kulp, S.K.; Lai, I.-L.; Hsu, E.-C.; He, W.A.; Frankhouser, D.E.; Yan, P.S.; Mo, X.; Bloomston, M.; Lesinski, G.B.; et al. Preclinical Investigation of the Novel Histone Deacetylase Inhibitor AR-42 in the Treatment of Cancer-Induced Cachexia. J. Natl. Cancer Inst. 2015, 107, djv274. [CrossRef]

59. Pertea, M.; Kim, D.; Pertea, G.M.; Leek, J.T.; Salzberg, S.L. Transcript-level expression analysis of RNA-seq experiments with HISAT, StringTie and Ballgown. Nat. Protoc. 2016, 11, 1650-1667. [CrossRef] [PubMed]

60. Li, H.; Handsaker, B.; Wysoker, A.; Fennell, T.; Ruan, J.; Homer, N.; Marth, G.; Abecasis, G.; Durbin, R. 1000 Genome Project Data Processing Subgroup. The Sequence Alignment/Map format and SAMtools. Bioinformatics 2009, 25, 2078-2079. [CrossRef]

61. Niknafs, Y.S.; Pandian, B.; Iyer, H.K.; Chinnaiyan, A.M.; Iyer, M.K. TACO produces robust multisample transcriptome assem-blies from RNA-Seq. Nat. Methods 2017, 14, 68-70. [CrossRef] [PubMed]

62. Love, M.I.; Huber, W.; Anders, S. Moderated estimation of fold change and dispersion for RNA-Seq data with DESeq2. Genome Biol. 2014, 15, 550. [CrossRef] [PubMed]

63. Wang, L.; Park, H.J.; Dasari, S.; Wang, S.; Kocher, J.-P.; Li, W. CPAT: Coding-Potential Assessment Tool using an alignment-free logistic regression model. Nucleic Acids Res. 2013, 41, e74. [CrossRef]

64. Zhou, Y.; Zhou, B.; Pache, L.; Chang, M.; Khodabakhshi, A.H.; Tanaseichuk, O.; Benner, C.; Chanda, S.K. Metascape provides a biologist-oriented resource for the analysis of systems-level datasets. Nat. Commun. 2019, 10, 1523. [CrossRef] [PubMed]

65. Li, T.; Chen, B.; Yang, P.; Wang, D.; Du, B.; Kang, L. Long non-coding RNA derived from lncRNA-mRNA co-expression net-works modulates the locust phase change. Genom. Proteom. Bioinform. 2020, in press. [CrossRef] [PubMed]

66. Reimand, J.; Isserlin, R.; Voisin, V.; Kucera, M.; Tannus-Lopes, C.; Rostamianfar, A.; Wadi, L.; Meyer, M.; Wong, J.; Xu, C.; et al. Pathway enrichment analysis and visualization of omics data using g: Profiler, GSEA, Cytoscape and EnrichmentMap. Nat. Protoc. 2019, 14, 482-517. [CrossRef] [PubMed] 